\title{
The prevalence and risk factors of work-related musculoskeletal disorders among adults in Ethiopia: a systematic review protocol
}

\section{Tsiwaye Gebreyesus}

Mekelle University College of Health Sciences

Kalkidan Nigussie

University of Gondar College of Medicine and Health Sciences

Moges Gashaw Getnet

University of Gondar College of Medicine and Health Sciences

Balamurugan Janakiraman ( $\sim$ bala77physio@gmail.com )

University of Gondar College of Medicine and Health Sciences https://orcid.org/0000-0003-3866-9351

\section{Protocol}

Keywords: Prevalence, Work-related musculoskeletal pain, Population-based observational studies, Systematic review, Ethiopia.

Posted Date: May 5th, 2020

DOl: https://doi.org/10.21203/rs.3.rs-17728/v2

License: (9) This work is licensed under a Creative Commons Attribution 4.0 International License. Read Full License

Version of Record: A version of this preprint was published at Systematic Reviews on June 8th, 2020. See the published version at https://doi.org/10.1186/s13643-020-01403-9. 


\section{Abstract}

Background: Work-related musculoskeletal disorders imposes a significant and most often underappreciated burden to the individual, nation, healthcare system, and society as a whole. A preliminary literature search suggests that there are at present no reliable estimates on the total prevalence of work-related musculoskeletal disorders in Ethiopia. Further, in Ethiopia, the cloud of infectious and other non-communicable diseases has led to a lack of attention towards work-related musculoskeletal disorders, empirical under-representation, and possible human capital loss. The objective of this protocol is to present a transparent process for how to review the existing literature on the prevalence rates and determinant factors of work-related musculoskeletal disorders in Ethiopia.

Method: The proposed methodology is based on Preferred Reporting Systematic Reviews and Protocols (PRISMA-P) statements on the conduct of systematic review and meta-analysis and the MOOSE guidelines for Meta-analysis and Systematic Reviews of Observational Studies. The electronic databases MEDLINE, PubMed, CINAHL, Science Direct index, SCOPUS, PEDro, PsyINFO, Embase, Ebsco, and Google Scholar will be systematically searched. Besides, the grey literature resources such as databases or websites of dissertations and theses will be searched. The reference list of screened articles will also be hand searched. All observational studies reporting on the prevalence of work-related musculoskeletal pain of any bodily region among adult Ethiopians will be included. Random and quality effects models will be used to calculate pooled prevalence with a $95 \%$ confidence interval. Subgroup and sensitivity analyses will be performed. Publication bias and heterogeneity between the included studies will also be assessed and reported.

Discussion: The proposed systematic review and meta-analysis will provide valid insight into the pooled prevalence of multi-regional work-related musculoskeletal pain and factors associated. The consensus of data from this review will surely help the policymakers in occupational health and health care sectors in identifying priority areas for interventions in work-related musculoskeletal disorders and will also serve as a baseline for the decision-making processes of musculoskeletal health promotion, work exposure implementations, and prevention programs in workplaces.

\section{Background}

Musculoskeletal disorders (MSDs) are common workplace health problems characterized by a range of symptoms like pain, ache, and discomfort in bodily regions [1,2]. Musculoskeletal disorders (MSDs) are impairments of body structures such as muscles, tendons, fascia, ligaments, joints, nerves, bones, or a localized blood circulation system either caused or aggravated by poor fitness, and poor health habits, but a major proportion of MSDs are caused by physical work exposures [3,4]. And, these impairment occurs secondary to sudden injuries and cumulative trauma, with the latter being the most common mechanism behind work-related musculoskeletal disorders (WRMSDs) $[1,3,5]$. 
According to Global Burden of Diseases (GBD), injuries, and risk factors study 2017, between 2007 and 2017, among the three causes which are observed to have resulted in a further increase in the number of all-age years lived with disability (YLDs), low back pain (LBP) is attributable to a further increase of $17 \cdot 5 \%,(95 \%$ UI 16.2-19.0), While the YLDs counts are heavily concentrated in working-age (i.e. from 20 -54 years), a pattern which is particularly evident among causes such as musculoskeletal disorders, mental disorders, and neurological disorders which sum to more than $45 \%$ of all YLDs in these age groups [6,7]. This finding is notable as the working age group in countries with low socio-demographic index (SDI) has a considerable number of years to live and musculoskeletal disorders of even lower to moderate disability weights, can cause substantial non-fatal burden and lost human capital [8]. Moreover, musculoskeletal disorders being three of the top 10 conditions in terms of disability and burden from non-communicable diseases (NCDs) in developing countries. Total disability-adjusted life years (DALYs) from low back and neck pain also increased, rising by more than 17\% from 2000 to 2017. [9-11].

GBD 2017 reported that MSD's are the second most common cause of years lost to disability, while years of life lost are declining in sub-Saharan. Further, the burden of MSD will only continue to increase as the sub-Saharan population ages [7,12]. Work-related musculoskeletal disorders (WRMSDs) and other musculoskeletal conditions remain less prioritized and empirically unrepresented in low-middle income countries (LMICs), particularly in Ethiopia due to focus on more pressing and life-threatening health issues like NCDs and infectious diseases [13]. A previous systematic review has however reported on the prevalence of WRMSDs in Africa which included a majority of studies from South Africa and Nigeria. The prevalence of WRMSDs varies from $13 \%$ to $92 \%$ in South Africa and Ghana respectively [14]. Empirically, racial, economic, and social homogeneity is not a feature of Africa and may underlie the reported difference in the prevalence. Hence, it is logical, therefore, to argue that genetic diversity, differences in social structure, economics, and other Ethiopian specific factors may influence the WRMSDs prevalence among Ethiopians. Further, musculoskeletal disorders remain a major global health concern and an immense burden for LMICs like Ethiopia where health budgets are already constrained and channeled towards the life-threatening conditions. Studies have shown that musculoskeletal pain prevalence among the working population in Ethiopia varies from $35 \%$ to $74.5 \%$ [13-20].

Though in the past decade many individual studies have reported the prevalence of regional pain, general WRMSDs and factors associated with the working Ethiopian population, to our knowledge, no published systematic review reporting on the prevalence of work-related musculoskeletal on Ethiopian adult population exists. The consensus of data from this review will help occupational health-related policymakers, health care professionals and program managers in developing countries in particular Ethiopia, to gain a better understanding of the prevalence, causes, and trends to build a better evidence-based occupational musculoskeletal health and disorders prevention programs. Therefore, the objectives of this systematic review and meta-analysis are to assess the relative prevalence of work-related musculoskeletal disorders, identifying the role of occupation and other factors associated with good or poor musculoskeletal health among the working adult population in Ethiopia. The review will aim to synthesize all possible available information for occupational health policymakers, health care workers, and identify priority areas for interventions in work-related musculoskeletal disorders in Ethiopia. 
Specific review questions are:

- What is the prevalence of musculoskeletal disorders in the categories of bodily regions, for different occupational settings and subgroups in Ethiopia according to internationally published studies?

- What are the occupational factors and patterns (pain sites, frequency, distribution across body) of musculoskeletal pain, what are the factors that cause and/or related to good or poor musculoskeletal health for different occupational settings and subgroups in the adult working population in Ethiopia according to internationally published studies?

- What is the methodological quality of the prevalence studies, intending to identify approaches to improve future research quality?

\section{Methods}

This systematic review and meta-analysis have been enrolled for registration at the International Prospective Register of Systematic Reviews and meta-analysis (PROSPERO). This review protocol adheres to the Preferred Reporting Items for Systematic Reviews and Meta-analysis Protocols (PRISMA-P, 2015) $[23,24]$ (Additional file 1) and the MOOSE guidelines for Meta-Analyses and Systematic Reviews of Observational Studies [25]. PRISMA flow diagram will be presented to describe the screening and selection processes. The findings of the review will be illustrated through figures and tables.

\section{Data sources and search strategies}

A systematic search of databases like MEDLINE, PubMed, CINAHL, Science Direct index, PEDro, PsyINFO, Embase, Ebsco, and Google Scholar will be conducted. Studies conducted in English and other languages with the English version will be included in the review. Both Medical Subject Headings (MeSH) and free text words will be used while searching for relevant articles. We will use the following search terms; "workrelated musculoskeletal disorders" [-MeSH], "musculoskeletal pain" [-MeSH], "musculoskeletal disorders" [-MeSH], neck pain, spinal pain, back pain, low back pain, shoulder pain, elbow pain, groin pain, knee pain, ankle pain in combination with "workers", "employees" "staffs", adults, "occupation", "workplace" "prevalence" and "Ethiopian", "AND" and "OR" Boolean operator terms will be used as appropriate. The search query and details like time, date of search, terms used, and the number of hits/results will be recorded for every session to keep a note new studies add during the review period. The details of electronic search terms and strategies are included in the supplementary information (Additional file 2). Furthermore, we will also search grey literature resources such as a database or website of dissertations and theses, google, WHO websites and websites of other professional bodies(eg; Centers for Disease Control and Prevention, Workplace Health Promotion). The reference lists of the included articles will be hand-searched for additional eligible studies. Moreover, the corresponding authors will be contacted by mail whenever a need arise or for any difficulties faced during data extraction.

\section{Inclusion and exclusion criteria}


All observational studies reporting on the prevalence of musculoskeletal disorders and/or musculoskeletal pain with factors associated with musculoskeletal disorders among the adult $(\geq 18$ years of age) working population of Ethiopia will be included. As we are primarily interested in the contemporary literature on WRMSDs in Ethiopia, we will examine publications from January 2000. The primary outcome will be to identify the point prevalence, period prevalence, prevalence rate of WRMSDs and the secondary outcome will be to identify risk and protective factors of WRMSDs among Ethiopian adults. Articles that meet the following criteria will be included: (1) Conducted solely among adult men or women Ethiopian those who work in governmental sectors or private sectors or self-employed, (2) reported the prevalence using standardized pain scales or questionnaires (eg: Standardized Nordic Questionnaire, Dutch Musculoskeletal questionnaire, or Visual Analogue Scale) providing sufficient information to calculate prevalence, and (3) Published in English language. Review, clinical commentary, conference abstracts, letter to editors, non-human articles and studies conducted outside of Ethiopia will be excluded. Studies published in non-English language will be excluded as we do not possess resources to pay or delegate volunteers to translate the data.

\section{Study screening and selection for inclusion in the review}

The titles and abstracts of articles retrieved from the search of different databases will be stored and managed in a Zotero version 5 reference manager and we will remove the duplicate records from it. Two reviewers (BJ and $\mathrm{KN}$ ) will independently review the titles and abstract part of all the articles, the full text of the potentially eligible article will be assessed for compliance and analyzed for inclusion.

Disagreements or conflicts throughout the review process will be resolved by consensus and if needed, by requesting the opinion of the third and fourth reviewers. The consistency of the selection process and quality assessment across the reviewers will be ensured by calculating the level of inter-rater agreement (Kappa statistics) [26].

\section{Data extraction and management}

Once eligible studies are identified, two independent reviewers will extract the data using a prepared standardized data extraction form. Reviewers will not perform data extraction from an article of which they were (co-) authors to eliminate possible bias. Data such as first author's last name, year of publication, study location within Ethiopia, sample size, response rate, the reason for non-response, number of events, regions of pain reported, data on prevalence, recall period, ascertainment of outcome measures, risk factor or protective factors determined by each study along with their respective odds ratio (OR) and 95\% confidence interval, and information needed for the risk of bias assessment will be extracted Table 1.

Table 1 Data items that will be extracted 


\begin{tabular}{|l|l|}
\hline 1. & $\begin{array}{l}\text { Publication details: Title, Journal, Author, Year, City/region in Ethiopia, where the study was conducted, Type } \\
\text { of publication, and Source of funding, if any. }\end{array}$ \\
\hline 2. & $\begin{array}{l}\text { Design: Type of study design (observational studies, cross-sectional, cohort, case-control, others); aims or } \\
\text { objective of the study, method of data collection, outcome variable (measures and operational definition), } \\
\text { response rate, recruitment and sampling method, eligibility (inclusion and exclusion criteria) }\end{array}$ \\
\hline 3. & $\begin{array}{l}\text { Study participants information's: Sample size, population characteristics including settings, role in the } \\
\text { institution, age, gender, ethnicity, education level, demographic information's, behavioral information's, } \\
\text { physical measurements, comorbidities, other health-related characteristics, Employment details, work and } \\
\text { work-environment related characteristics, bodily distribution of pain }\end{array}$ \\
\hline 4. & $\begin{array}{l}\text { Data for outcome variables: all reported estimates, or sufficient data to calculate an estimate of the point } \\
\text { prevalence, cumulative incidence, and incidence rate of musculoskeletal disorders in categories of bodily } \\
\text { distribution like low back pain, neck pain, shoulder pain, elbow pain, wrist and hand pain, hip pain, knee } \\
\text { pain, ankle pain, etc }\end{array}$ \\
\hline 5. & $\begin{array}{l}\text { Limitations: Bias (selection, response, information), limitation of outcome measures/tools, and limitation } \\
\text { reported by authors }\end{array}$ \\
\hline
\end{tabular}

\section{Risk of bias and quality assessment}

Two review authors (BJ and $\mathrm{KN}$ ) will independently assess the quality of all included studies using the Newcastle-Ottawa Quality Assessment tool adapted for cross-sectional studies $[27,28]$. The tool will be further adapted for use in this review (Additional file 3). Discrepancies of aggregate or total scores will be resolved by the third reviewer (TG) after a detailed evaluation of the source of the discrepancy. The tool contains three domains; selection of participants (3 items), quality of data (4 items), and definition of work-related musculoskeletal disorders (3 items). For the purpose of this review, all the items in the appraisal tool will be equally weighted and so the total score will be 10 . There will be no subminimum score criteria for inclusion of studies.

\section{Data synthesis}

The prevalence rate, the logarithm of prevalence, and standard error (SE) of the logarithm of prevalence will be computed. Correspondingly, for the factors associated, the logarithm of OR and SE of the logarithms of OR will be calculated. The pooled prevalence (proportion) of musculoskeletal disorders or musculoskeletal pain-related work and the pooled odds ratios (OR) of associated factors with a $95 \%$ confidence interval will be calculated using random-effects and quality effects models. The qualityeffects meta-analysis [29] will be used to examine how the quality of each study influenced the pooled estimate compared with the results from the random effects [30]. The quality scores of each included study will be incorporated in the calculation of study weight to improve the robustness and help minimize the estimator variance and subjectivity in quality assessment. The presence of heterogeneity among studies will be examined using both Cochrane's Q statistics and the $P^{2}$ statistics. $P^{2}$ values greater than $50 \%$ will be declared as the presence of substantial heterogeneity [31,32]. Funnel plots will be used to 
assess publication bias[33] and in addition Egger's regression test $(p<0.05)$ [34] and Beggs equations will be computed to declare publication bias. Double arsine transformation will be used in the case of variance instability [35].

Possible subgroups will be identified based on the study characteristics and population characteristics. Subgroup analysis will be performed to determine the source of heterogeneity attributed to gender, sample size, place of study, study setting, year of publication, outcome tool used, type of occupation, and region of pain. Sensitivity analysis will be performed after excluding each study one by one and the pooled estimate will be calculated for the remaining studies. All statistical analyses will be performed using Meta XL version 5.3 [36] and STATA 15 Metaprop package [37].

\section{Timeline, presenting and reporting of the results}

The review process will commence only after the final peer-review comments are received, and the protocol accepted for publication. The study selection step by step process will be outlined in a flow diagram and the reasons for exclusion will also be mentioned. The study characteristics, risk of bias, and quality assessment of the included studies will be presented in tables. Forest plots will be used to display the pooled estimates of prevalence proportions.

\section{Discussion}

In the past decade, institutional and population-based studies on work-related musculoskeletal disorders have grown in Ethiopia. This proposed review aims to provide a current overview of the prevalence of musculoskeletal disorders in working-aged Ethiopian adults from various occupations, cultures, and age groups to provide a better understanding of musculoskeletal health and its effect on their life.

Furthermore, this will be the first systematic review and meta-analysis that will determine the pooled prevalence of musculoskeletal disorders among the adult working population in Ethiopia. In addition, this paper will also identify factors associated with work-related musculoskeletal disorders among Ethiopians. Though WRMSD is not life-threatening, initiatives to include WRMSDs is critical in developing countries rather than being stand-alone. We believe that the inclusion of WRMSDs in Ethiopia will avoid efforts doubling and wasting of resources in the future. The rationale for the need to conduct this review is the previous two African based reviews reporting on WRMSDs (2015) and low back pain (2007) were limited by not including any studies from Ethiopia $[14,38]$ and in addition, Ethiopian-specific factors like social structure, genetic diversity, nutritional status, and work culture will surely add more insight on the Ethiopian working population.

Among the potential challenges we anticipate are most importantly at review level decisions as to studies reporting the prevalence of headache should be classified as dealing with musculoskeletal disorders or not, as well as at study level by heterogeneity in occupations, measurement tools, and region of pain. We will strive to mitigate these anticipated and future challenges by highlighting decision criteria, limitations, gaps in knowledge, and caution in the interpretation of findings. 


\section{Strengths and Conceivable limitations}

The strengths of this review include established national-level consensus of data, as well as a systematic and transparent approach. Our search will be conducted in close collaboration with the specialized research librarian, the screening and extraction will be performed by two researchers using a standardized extraction form. The inter-rater agreement between reviewers in the selection process will be statistically assessed. Furthermore, this review will also be the first one to consensus data on work-related musculoskeletal disorders in Ethiopia.

We anticipate that publication bias and heterogeneity may pose a limitation for this review. There are also possibilities of reporting and response set bias. In addition, this study is restricted to published reports in the English language.

\section{Dissemination}

The findings of this review will be disseminated through publication in peer-reviewed journals and conference presentations at the relevant venue.

\section{List Of Abbreviations}

BoD - Burden of Diseases, DALYs - Disability-adjusted life years, GBD - Global Burden of Disease, LBP Low Back Pain, LMICs - Low-middle income countries, MeSH - Medical Subject Headings, MOOSE Meta-analysis Of Observational Studies in Epidemiology, MSDs - Musculoskeletal Disorders, NCD - NonCommunicable Diseases, OR - Odds Ratio, PRISMA-P - Preferred Reporting Items for Systematic Reviews and Meta-analysis Protocols, SDI - Socio-demographic index, SE - Standard Error, WHO - World Health Organization, WRMSDs - Work-Related Musculoskeletal Disorders, YLD - Years lived with disability

\section{Declarations}

\section{Ethical approval and consent to participate:}

Not required

\section{Consent for publication:}

Not applicable

\section{Data availability:}

Not applicable

\section{Competing interests:}

The authors declare no competing interests 


\section{Funding:}

The authors have not declared a specific grant for this review from any funding organization or agency.

\section{Author contributions}

TG conceived the research question, edited the protocol, and designed the study, MG, KN and BJ designed the search strategy, will participate in the search process, appraise the quality of the articles, and extract needed data independently. TG will analyze and interpret the results. All authors read and approve this protocol before sending it for the publication. BJ will be the guarantor, supervisor, and corresponding person on behalf of all the reviewers.

\section{Acknowledgments:}

We thank Mekelle University, School of Medicine and University of Gondar, School of Medicine for their help in access to University subscribed web databases.

\section{Authors information}

TG is working as a Lecturer of Physiotherapy, School of Medicine, College of Medicine and Health Sciences, Mekelle University, Mekelle, Ethiopia. BJ Assistant professor of Physiotherapy \& Senior clinical consultant physiotherapist and MG, KN lecturer in Physiotherapy, Senior Physiotherapist, School of Medicine, College of Medicine and Health Sciences and specialized hospital, University of Gondar, Gondar, Ethiopia

\section{References}

1. Al-Hourani Z, Nazzal M, Khader Y, Almhdawi K, Bibars AR. Work-related musculoskeletal disorders among Jordanian dental technicians: Prevalence and associated factors. Work. 2017;56(4):617-23.

2. Bethge M. [Work-Related Medical Rehabilitation]. Rehabilitation (Stuttg). 2017 Feb;56(1):14-21.

3. Roquelaure Y, Bodin J, Descatha A, Petit A. [Musculoskeletal disorders: how to recognize them as occupational diseas]. Rev Prat. 2018 Dec;68(10):1132-4.

4. Gómez-Galán M, Pérez-Alonso J, Callejón-Ferre Á-J, López-Martínez J. Musculoskeletal disorders: OWAS review. Ind Health. 2017 Aug 8;55(4):314-37.

5. Oranye NO, Bennett J. Prevalence of work-related musculoskeletal and non-musculoskeletal injuries in health care workers: the implications for work disability management. Ergonomics. 2018;61(3):355-66.

6. Safiri S, Kolahi A-A, Hoy D, Buchbinder R, Mansournia MA, Bettampadi D, et al. Global, regional, and national burden of neck pain in the general population, 1990-2017: systematic analysis of the Global Burden of Disease Study 2017. bmj. 2020;368. 
7. Vos T, Allen C, Arora M, Barber RM, Bhutta ZA, Brown A, et al. Global, regional, and national incidence, prevalence, and years lived with disability for 310 diseases and injuries, 1990-2015: a systematic analysis for the Global Burden of Disease Study 2015. The Lancet. 2016;388(10053):1545-602.

8. Human Capital: The Greatest Asset of Economies on the Rise [Internet]. World Bank. [cited 2020 Apr 16]. Available from: https://www.worldbank.org/en/news/opinion/2017/04/03/human-capital-thegreatest-asset-of-economies-on-the-rise

9. Hoy DG, Smith E, Cross M, Sanchez-Riera L, Buchbinder R, Blyth FM, et al. The global burden of musculoskeletal conditions for 2010: an overview of methods. Annals of the rheumatic diseases. 2014;73(6):982-9.

10. Hoy DG, Smith E, Cross M, Sanchez-Riera L, Blyth FM, Buchbinder R, et al. Reflecting on the global burden of musculoskeletal conditions: lessons learnt from the global burden of disease 2010 study and the next steps forward. Annals of the rheumatic diseases. 2015;74(1):4-7.

11. Storheim K, Zwart J-A. Musculoskeletal disorders and the Global Burden of Disease study. 2014;

12. Fink DL, Oladele D, Etomi O, Olaosebikan H, Dey ID, Adelowo OO. We must harness technology to deliver the musculoskeletal disease epidemiology that is urgently needed across sub-Saharan Africa. Clinical rheumatology. 2018;37(5):1439-40.

13. Woolf AD, Brooks P, Åkesson K, Mody GM. Prevention of musculoskeletal conditions in the developing world. Best Practice \& Research Clinical Rheumatology. 2008;22(4):759-72.

14. Wanyonyi N, Frantz J. Prevalence of work-related musculoskeletal disorders in Africa: a systematic review. Physiotherapy. 2015;101:e1604-5.

15. Temesgen MH, Belay GJ, Gelaw AY, Janakiraman B, Animut Y. Burden of shoulder and/neck pain among school teachers in Ethiopia. BMC musculoskeletal disorders. 2019;20(1):18.

16. Kebede Deyyas W, Tafese A. Environmental and organizational factors associated with elbow/forearm and hand/wrist disorder among sewing machine operators of garment industry in Ethiopia. Journal of environmental and public health. 2014;2014.

17. Dheresa M, Geda B. Low Back Pain and Associated Factors among Nurses Working in Public Hospitals of Harari region and Dire Dawa City Administration, Eastern Ethiopia. 2018;

18. Yosef T, Belachew A, Tefera Y. Magnitude and Contributing Factors of Low Back Pain among Long Distance Truck Drivers at Modjo Dry Port, Ethiopia: A Cross-Sectional Study. Journal of environmental and public health. 2019;2019.

19. Mekonnen TH. Work-Related Factors Associated with Low Back Pain Among Nurse Professionals in East and West Wollega Zones, Western Ethiopia, 2017: A Cross-Sectional Study. Pain and therapy. 2019;8(2):239-47.

20. Wami SD, Dessie A, Chercos DH. The impact of work-related risk factors on the development of neck and upper limb pain among low wage hotel housekeepers in Gondar town, Northwest Ethiopia: institution-based cross-sectional study. Environmental health and preventive medicine. 2019;24(1):27. 
21. Tafese A, Nega A, Kifle M, Kebede W. Predictors of occupational exposure to neck and shoulder musculoskeletal disorders among sewing machine operators of garment industries in Ethiopia. Science Journal of Public Health. 2014;2(6):577-83.

22. Abraha TH, Demoz AT, Moges HG, Ahmmed AN. Predictors of back disorder among Almeda textile factory workers, North Ethiopia. BMC research notes. 2018;11(1):304.

23. Moher D, Shamseer L, Clarke M, Ghersi D, Liberati A, Petticrew M, et al. Preferred reporting items for systematic review and meta-analysis protocols (PRISMA-P) 2015 statement. Systematic reviews. 2015;4(1):1.

24. Shamseer L, Moher D, Clarke M, Ghersi D, Liberati A, Petticrew M, et al. Preferred reporting items for systematic review and meta-analysis protocols (PRISMA-P) 2015: elaboration and explanation. Bmj. 2015;349:g7647.

25. Stroup DF, Berlin JA, Morton SC, Olkin I, Williamson GD, Rennie D, et al. Meta-analysis of observational studies in epidemiology: a proposal for reporting. Jama. 2000;283(15):2008-12.

26. Cohn J. Statistical power analysis for the behavioral sciences. Lawrence Earlbam Associates, Hillsdale, NJ. 1988;

27. Luchini C, Stubbs B, Solmi M, Veronese N. Assessing the quality of studies in meta-analyses: Advantages and limitations of the Newcastle Ottawa Scale. World J Meta-Anal. 2017;5(4):80-4.

28. https:// wellcomeopenresearch.s3.amazonaws.com/supplementary/13880/ea30a2fba15a-44a9b35e-5f0914db80b3.docx]. Accessed 1 Feb 2019. - Google Search [Internet]. [cited 2020 Jan 11].

29. Doi SA, Thalib L. A quality-effects model for meta-analysis. Epidemiology. 2008;94-100.

30. Berkey CS, Hoaglin DC, Mosteller F, Colditz GA. A random-effects regression model for meta-analysis. Statistics in medicine. 1995;14(4):395-411.

31. Higgins JP, Thompson SG. Quantifying heterogeneity in a meta-analysis. Statistics in medicine. 2002;21(11):1539-58.

32. Stroup DF, Berlin JA, Morton SC, Olkin I, Williamson GD, Rennie D, et al. Meta-analysis of observational studies in epidemiology: a proposal for reporting. Jama. 2000;283(15):2008-12.

33. Liu JL. The role of the funnel plot in detecting publication and related biases in meta-analysis. Evidence-based dentistry. 2011;12(4):121.

34. Sterne JA, Egger M. Regression methods to detect publication and other bias in meta-analysis. Publication bias in meta-analysis: Prevention, assessment and adjustments. 2005;99-110.

35. Barendregt JJ, Doi SA, Lee YY, Norman RE, Vos T. Meta-analysis of prevalence. J Epidemiol Community Health. 2013;67(11):974-8.

36. Barendregt J, Doi S. MetaXL user guide version 5.3. EpiGear International Pty Ltd. 2016;

37. Nyaga VN, Arbyn M, Aerts M. Metaprop: a Stata command to perform meta-analysis of binomial data. Archives of Public Health. 2014;72(1):39.

38. Louw QA, Morris LD, Grimmer-Somers K. The prevalence of low back pain in Africa: a systematic review. BMC Musculoskeletal disorders. 2007;8(1):105. 


\section{Additional Files}

Additional file 1: PRISMA-P (Preferred Reporting Items for Systematic review and Meta-Analysis Protocols) 2015 checklist: recommended items to address in a systematic review protocol.

Additional file 2: Example search that will be used for screening of articles in PubMed database.

Additional file 3: Adapted table of Newcastle-Ottawa quality assessment tool

\section{Supplementary Files}

This is a list of supplementary files associated with this preprint. Click to download.

- Additionalfile1.docx

- Additionalfile3.docx

- Additionalfile2.docx 